\title{
First-Principles Investigation of Bilayer Fluorographene
}

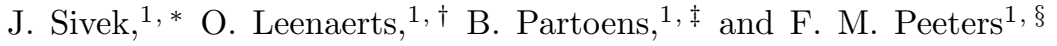 \\ ${ }^{1}$ Departement Fysica, Universiteit Antwerpen, Groenenborgerlaan 171, B-2020 Antwerpen, Belgium
}

(Dated: October 7, 2018)

\begin{abstract}
$A b$ initio calculations within the density functional theory formalism are performed to investigate the stability and electronic properties of fluorinated bilayer graphene (bilayer fluorographene). A comparison is made to previously investigated graphane, bilayer graphane, and fluorographene. Bilayer fluorographene is found to be a much more stable material than bilayer graphane. Its electronic band structure is similar to that of monolayer fluorographene, but its electronic band gap is significantly larger (about $1 \mathrm{eV}$ ). We also calculate the effective masses around the $\Gamma$-point for fluorographene and bilayer fluorographene and find that they are isotropic, in contrast to earlier reports. Furthermore, it is found that bilayer fluorographene is almost as strong as graphene, as its 2D Young's modulus is approximately $300 \mathrm{~N} \mathrm{~m}^{-1}$.

PACS numbers: 61.48.Gh, 68.43.-h, 68.43.Bc, 68.43.Fg, 73.21.Ac, 81.05.Uw
\end{abstract}

\section{INTRODUCTION}

Since the first reports on the successful isolation of stable two-dimensional crystals consisting of a single atom layer by Novoselov et al. in 2004, [1, 2, researchers have been looking for ways to employ these new materials in real-world applications. Of particular interest is graphene, a single-layer derivative of graphite. Graphene attracts a lot of attention with its high crystal quality and very promising electronic properties. 3]

Despite its unique characteristics that might be exploited in future applications, 44 there exists a serious obstacle to use graphene in electronics as we know it today. Graphene is a zero-gap semiconductor, and the absence of a band gap is protected by inversion and timereversal symmetry. 8] Several approaches have been used or proposed to open and control a band gap in graphene and graphene derivatives: (i) mechanical modification, such as cutting the graphene sample into nanoribbons, 9, 10, (ii) application of a potential difference over a bilayer of graphene, 11 13 and finally, (iii) chemical modification that confines the $p_{z}$ electrons into covalent bonds. 14-18 The last approach includes the adsorption of radicals, such as oxygen, hydrogen, and fluorine atoms, on the surface of graphene that form chemical bonds with the carbon atoms and change their hybridization from $\mathrm{sp}^{2}$ to $\mathrm{sp}^{3}$. The chemical derivatives of graphene preserve the two-dimensional character of graphene, but they have vastly different electronic properties. This makes them possibly more interesting materials for applications in electronics than intrinsic graphene.

Among the possible derivatives of graphene, there are two that have attracted special attention, namely, graphane and fluorographene (graphene fluoride), which

\footnotetext{
*Electronic address: jozef.sivek@ua.ac.be

${ }^{\dagger}$ Electronic address: ortwin.leenaerts@ua.ac.be

${ }^{\ddagger}$ Electronic address: bart.partoens@ua.ac.be

$\S$ Electronic address: francois.peeters@ua.ac.be
}

result from the hydrogenation and fluorination of graphene, respectively. They are theoretically predicted [19-26 and experimentally observed 14, 16, 18, 27, 28, to form crystalline materials, in contrast to, for example, graphene oxide. 29, 30.

These new two-dimensional crystals are currently the subject of a considerable amount of experimental and theoretical work. However, the process of chemical modification is not restricted to monolayer graphene. It has been proposed that this process can be expanded to bilayer graphene as well. 31 Ab initio calculations showed that the weak van der Waals forces between the graphene layers are replaced by much stronger covalent bonds that stabilize the structure and that, at full coverage, a bilayer analogue of graphane is formed. The electronic structure of intrinsic monolayer and bilayer graphane are very similar, 32 but their mechanical/elastic properties are expected to be substantially different.

The difference in elastomechanical qualities can be an important issue. It was recently demonstrated 33] that the roughness of monolayer graphane surfaces is considerably larger than that of graphene. This increase of the size of the ripple formation can be explained by the lower stiffness of graphane [34] and its different vibrational properties as compared with graphene. 35] One way to reduce the increased roughness is to consider bilayers instead of single layers. As is well known from experiment, the ripple formation in bilayer graphene is strongly reduced; that is, they are removed by the interlayer interaction. 36. A similar effect can be expected for bilayer graphane where the interlayer interaction is even more important.

In this paper, we investigate whether it is also possible to fluorinate bilayer graphene. We perform ab initio calculations to determine the stability and structural properties of a fluorinated bilayer of graphene, hereafter called bilayer fluorographene (for a better notion of the chemical composition and the structure, see Fig. 11). We find that bilayer fluorographene is a much more stable compound than bilayer graphane, although more fluorination is needed to induce interlayer covalent $\mathrm{C}-\mathrm{C}$ bonds. The 
structural properties of bilayer fluorographene are in between those of monolayer fluorographene and diamond; other characteristics are closer to those of initial fluorographene.

This paper is organized as follows: First, we describe the computational details of our $a b$ initio calculations, followed by an investigation of the stability and the formation conditions of interlayer covalent $\mathrm{C}-\mathrm{C}$ bonds. Next, the overall stability and the geometrical properties of fully fluorinated bilayer graphene (i.e., bilayer fluorographene) are examined. These properties are subsequently compared with those of diamond and fluorographene. To conclude, we investigate the electronic band structure and band gaps of single-layer and bilayer fluorographene, together with the effective masses of the various possible charge carriers and elastic properties represented by their 2D Young's moduli.

\section{CALCULATIONS}

All our calculations were done within the density functional theory (DFT) formalism as implemented in the VASP package with usage of the local density approximation (LDA) and the Perdew, Burke, and Ernzerhof 37. generalized gradient approximation (GGA) for the exchange-correlation functional. We made use of the projector augmented wave method [38] and a plane-wave basis set with an energy cutoff of $500 \mathrm{eV}$. The relaxation of atomic positions was performed with forces smaller than $0.01 \mathrm{eV}^{-1}$.

Three types of supercells were used in our calculations: $3 \times 3$ and $2 \times 2$ supercells to study the adsorption properties of fluorine on a graphene bilayer for different concentrations and configurations of fluorine and a $1 \times 1$ unit cell for the calculation of the properties and electronic band structure of fully fluorinated graphene and bilayer graphene.

The sampling of the Brillouin zone was done for the different supercells with the equivalent of a $24 \times 24 \times 1$ Monkhorst-Pack 39 $k$-point grid for the monolayer or bilayer graphene unit cell (containing two carbon atoms per layer). Spin polarization was not included in the calculations because fluorination is not expected to induce magnetism in graphene. 40]

Because periodic boundary conditions were applied in all three dimensions, the height of the supercell was set to $20 \AA$ to include enough vacuum to minimize the interaction between adjacent layers. Additionally, we have performed a convergence test with respect to the planar size of the supercell. We obtain an accuracy in binding energies of less than $0.1 \mathrm{eV}$ and in bond lengths of less than $0.01 \AA$ with the chosen $3 \times 3$ supercell.

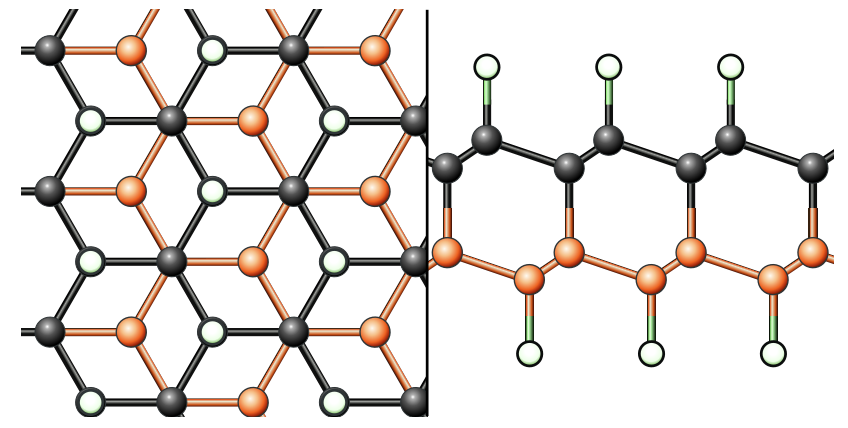

FIG. 1: Top (left) and side (right) view of bilayer fluorographene. The carbon atoms of the two layers are given by different colors (shades) for clarity and the fluorine atoms are in light green (open circles with the smallest diameter).

\section{RESULTS AND DISCUSSION}

\section{A. Fluorination of Bilayer Graphene}

The main objective of this paper is to investigate chemisorption of fluorine atoms on both sides of bilayer graphene. Hydrogenation and fluorination of the carbon atoms of graphene change their hybridization from $\mathrm{sp}^{2}$ to $\mathrm{sp}^{3}$. In the case of hydrogenation of bilayer graphene, it was demonstrated that stable interlayer $\mathrm{C}-\mathrm{C}$ bonds are induced at high concentrations of adsorbates. 31] In this section, we perform a similar study for the case of fluorination and highlight the similarities and differences as compared to hydrogenated bilayer graphene. We make use of the local density approximation, because this approximation gives a better description of the interlayer interaction in bilayer graphene over the generalized gradient approximation. 41]

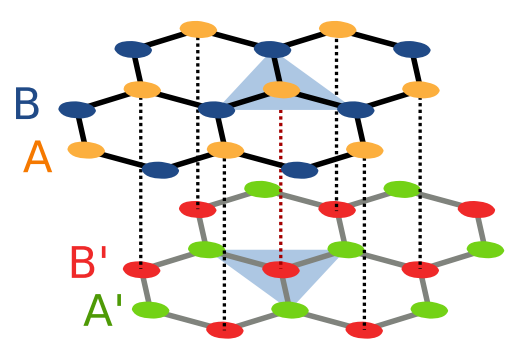

FIG. 2: Bilayer of AB-stacked graphene with the four different sublattices indicated by different colors. The A and B' sublattices are situated on top of each other, as illustrated by the dotted lines. The carbon atoms at the corners of the light triangles indicate the six neighbors to be fluorinated.

We only consider chemisorption on bilayer graphene with $\mathrm{AB}$ stacking because this is the most energetically favorable way of stacking graphene layers. As indicated in Fig. 2, this gives rise to four sublattices (two in each layer) of which two, namely, the A and B' sublattice, are exactly aligned on top of each other while the sublattices 
$\mathrm{B}$ and $\mathrm{A}^{\prime}$ are not. Since the adsorbates under investigation always chemisorb on top of a single $\mathrm{C}$ atom, there are two (inequivalent) adsorption sites, namely, on the A (or B') sublattice and on the B (or A') sublattice. It was found in an earlier study of hydrogen adsorption on bilayer graphene that there is a difference in adsorption energy of approximately $0.03 \mathrm{eV}$ in favor of the B sublattice. 31 The $\mathrm{H}$ atoms are, therefore, likely to attach to those carbon atoms that have no direct neighbors in the other layer. For $\mathrm{F}$ atoms, we find the same preference for adsorption on the B sublattice. However, the difference in adsorption energy for the two sublattices is only 0.3 meV, which is about 100 times smaller than in the case of hydrogen. This is caused by the different nature of $\mathrm{C}-\mathrm{F}$ bonds as compared with $\mathrm{C}-\mathrm{H}$ bonds: $\mathrm{C}-\mathrm{H}$ bonds are purely covalent, but $\mathrm{C}-\mathrm{F}$ bonds have a partially ionic character because of the large difference in electronegativity between $\mathrm{C}$ and $\mathrm{F}$.

The formation of a chemical bond between an adsorbate and a $\mathrm{C}$ atom of the bilayer of graphene induces a change in the hybridization of this $\mathrm{C}$ atom from $\mathrm{sp}^{2}$ to $\mathrm{sp}^{3}$. If the concentration of attached adsorbates on the preferred sublattice increases, the carbon atoms of the other sublattice also change their hybridization to $\mathrm{sp}^{3}$. This allows for the formation of covalent bonds between the two graphene layers. These interlayer bonds are formed between carbon atoms of the A and B' sublattices that are pushed to one another because of the changed hybridization of their neighbors.

For a single interlayer $\mathrm{C}-\mathrm{C}$ bond, there are six neighbors: three atoms in the $\mathrm{B}$ and three in the A' sublattice (Fig. 2). However, it is not necessary for all of these neighbors to change their hybridization in order for the interlayer bond to be formed, as in the case of hydrogen chemisorption on bilayer graphene, where only four of the neighboring carbon atoms need to be hydrogenated to induce a covalent interlayer bond. 31]

To study the formation of interlayer bonds upon fluorination in more detail, we gradually increase the number of attached fluorine atoms on neighboring carbon atoms from one to six. We completely relax the resulting systems and calculate the average and minimum distances between the $\mathrm{C}$ atoms of the two layers. The results of these computations are summarized in Table I From this table, a gradual decrease of the interlayer distance can be seen upon increasing the concentration of $\mathrm{F}$ atoms. At higher concentrations, however, a substantial jump in the minimum distance, $d_{\min }$, occurs when going from four to five fluorinated neighbors. This jump is about $1 \AA$ in size and clearly indicates the formation of an interlayer bond. It is thus necessary to fluorinate five of the six neighbors to induce an interlayer bond, in contrast to hydrogenation, where four hydrogenated neighbors suffice.

Additional information about the stability of the interlayer bond can be obtained by examining the formation and binding energy of the system at different concentrations of adsorbed $\mathrm{F}$ atoms. We define the formation energy $\left(E_{\mathrm{f}}\right)$ as the energy per attached fluorine atom
TABLE I: Interlayer chemical bond formation in a $3 \times 3$ supercell. The binding energy per $\mathrm{F}$ atom $\left(E_{\mathrm{b}}\right)$, the formation energy per $\mathrm{F}$ atom $\left(E_{\mathrm{f}}\right)$ and the average $\left(d_{\mathrm{avg}}\right)$ and minimum distance $\left(d_{\min }\right)$ between the $\mathrm{C}$ atoms of the two layers are shown for different number of adsorbed fluorine atoms and different configurations. Presence of interlayer bond is marked as chemical bond. Energies are given in $\mathrm{eV}$ and distances in $\AA$.

\begin{tabular}{cllllllc}
\hline \hline $\begin{array}{c}\text { no. } \\
\text { F atoms }\end{array}$ & configuration & $E_{\mathrm{b}}$ & $E_{\mathrm{f}}$ & $d_{\mathrm{avg}}$ & $d_{\min }$ & $\begin{array}{c}\text { chemical } \\
\text { bond }\end{array}$ \\
\hline 1 & B & -2.525 & -0.819 & 3.326 & 3.256 & no \\
2 & BB & -2.405 & -0.700 & 3.326 & 3.190 & no \\
2 & BA' & -2.555 & -0.850 & 3.213 & 3.085 & no \\
3 & BBB & -2.290 & -0.585 & 3.332 & 3.126 & no \\
3 & BBA' & -2.487 & -0.782 & 3.176 & 2.979 & no \\
4 & BBBA' & -2.384 & -0.679 & 3.150 & 2.903 & no \\
4 & BBA'A' & -2.479 & -0.773 & 3.061 & 2.821 & no \\
5 & BBBA'A' & -2.450 & -0.745 & 2.678 & 1.757 & yes \\
6 & BBBA'A'A' & -2.560 & -0.854 & 2.598 & 1.737 & yes \\
\hline \hline
\end{tabular}

with respect to intrinsic bilayer graphene and the diatomic molecule $\mathrm{F}_{2}$. The binding energy $\left(E_{\mathrm{b}}\right)$, on the other hand, is defined as the energy per fluorine atom (or $\mathrm{CF}$ pair) with respect to intrinsic bilayer graphene and atomic fluorine. Both energies are given in Table $\mathrm{I}$.

The formation energy, $E_{\mathrm{f}}$, is negative in all cases, which means that all the configurations are stable against molecular desorption from the graphene surface. This should be contrasted to the same concentrations of hydrogen atoms on the surface of bilayer graphene, where the energy becomes negative only in the case of almost fully hydrogenated bilayer graphene. 31. The value of the formation energies in the case of fluorination is significantly lower (almost $1 \mathrm{eV}$ ) than in the case of hydrogen chemisorption. This can be attributed to the weaker bond in the $\mathrm{F}_{2}$ as compared with the $\mathrm{H}_{2}$ molecule and is similar to the previously studied case of monolayer graphane and fluorographene. 23] The calculated binding energies also show that it is energetically favorable for the fluorine atoms to attach themselves on both sides of the bilayer: for the same number of chemisorbed $\mathrm{F}$ atoms, the configuration in which these atoms are distributed as equally as possible between the two layers is lower in energy and thus more stable. The fact that adsorbed $\mathrm{F}$ atoms on one side of the bilayer make it favorable for other $\mathrm{F}$ atoms to adsorb on the other side increases the chance of interlayer bond formation. Because these bonds are stable, we can imagine this process of fluorination and interlayer bond formation to continue until a fully covered bilayer of graphene fluoride is formed.

To test if the aforementioned interlayer bond creation scenario is truly energetically favorable, we considered a $2 \times 2$ supercell for a gradual fluorination with different configuration patterns. To be able to choose from the large number of different possible configurations, we need some guidelines for further investigation. As a first 
guideline, we distribute the $\mathrm{F}$ atoms equally on both sides of the bilayer because it is more energetically favorable, as discussed above. Four prevalent configurations are known for the fluorination of graphene: chair, boat, zigzag, and armchair. The chair configuration can be readily extended to the fluorination of bilayer graphene discussed above. The remaining three configurations share as a common feature that they contain dimers, as depicted in the inset of Fig. 3. Those dimers are used as the second guideline to reduce configuration space. The dimer $a$ is composed of $\mathrm{F}$ atoms bonded with directly neighboring $\mathrm{C}$ atoms, whereas the dimer $b$ consists of $\mathrm{F}$ atoms bonded with distant $\mathrm{C}$ atoms on the opposite sides of the hexagonal ring. For higher fluorine concentrations (above two dimers, one at each side of the bilayer), dimers $a$ and $b$ are combined and create a trimer configuration, as depicted in the inset $\mathrm{c}$ of Fig. 3.

The calculated results are given in Fig. 3 . In this figure, we present the formation energy per adatom for the different fluorination pathways. These results show that, for low concentrations of $\mathrm{F}$ atoms, the dimer $b$ configuration is more stable (carbon atoms from both sublattices are fluorinated, and no interlayer bonds are created). However, higher concentrations of $\mathrm{F}$ atoms are not feasible with the dimer configurations as the formation energy increases. However, if only the B and A' sublattices are fluorinated, the formation energy can decrease even more, finally creating bilayer fluorographene, with completely saturated $\mathrm{C}$ atoms, as can be seen in Fig. 1. It is also important to point out that the total formation energy for all adsorbed $\mathrm{F}$ atoms (not shown in the figure) is a monotonically decreasing function of the $\mathrm{F}$ atom concentration, and therefore, the local minimum for dimer $b$ will not result in an interruption of the adsorption.

Our calculations also showed that, for both dimer configurations, no interlayer bonding was formed. The dimers $a$ and $b$ transform for higher coverage into the trimer configuration, as indicated by the connection of the plots. For the adsorption on B and A' sublattices, the interlayer bond is created already with only four $\mathrm{F}$ atoms. This apparently different result from the previous $3 \times 3$ supercell calculations is due to the choice of the size of supercell. Four adatoms in a $2 \times 2$ supercell correspond to a higher percentage of $\mathrm{F}$ per $\mathrm{C}$ atoms than five adatoms in the $3 \times 3$ supercell.

We also performed calculations for the case when the A and B' sublattices, lying on top of each other, were fluorinated. As already mentioned, the preference for B (A') over A (B') sublattice for a single adatom is very small and unlikely to conduct the adsorption pattern. With increased $\mathrm{F}$ atom coverage, the formation energy per adatom for fluorination of the A and B' sublattice was found to be an increasing function of the total coverage. This results in a very unfavorable alternative over fluorination of B and A' sublattices.

From the calculations on the $\mathrm{F}$ adsorption in a $2 \times 2$ supercell, we can conclude that the chair configuration is the lowest-energy configuration, the only configuration that leads to interlayer bonding and in which a high fluorine concentration can be reached.

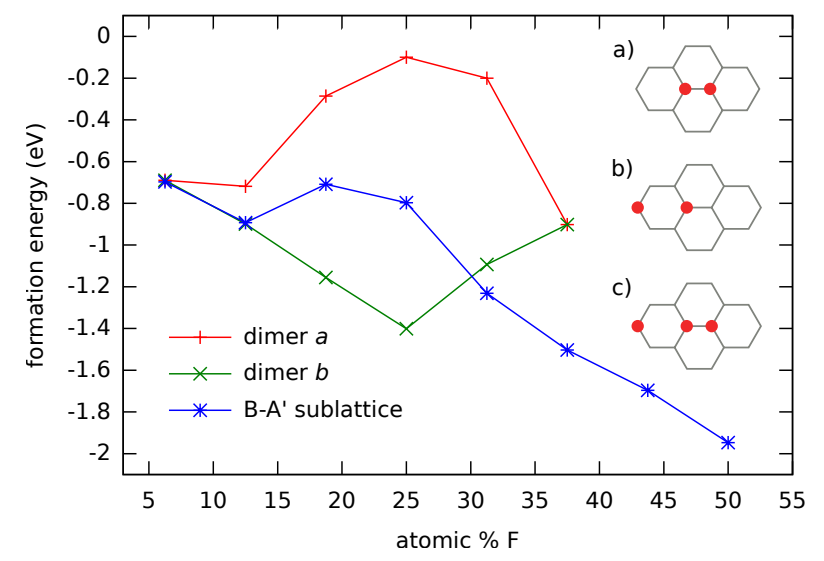

FIG. 3: Gradual fluorination of a $2 \times 2$ supercell of bilayer graphene. For increasing $\mathrm{F}$ concentration the formation energy per fluorine atom is shown. The curves show different distributions of the $\mathrm{F}$ atoms: according to dimer configuration $a$ (inset $\mathrm{a}$ ) and $b$ (inset b), only on sublattices B and A', and the placement of the $\mathrm{F}$ atoms only on sublattices $\mathrm{A}$ and $\mathrm{B}$ '. Dimers $a$ and $b$ can not be preserved for higher levels of fluorination; instead they transform into the trimer configuration (inset c).

\section{B. Properties of Bilayer Fluorographene}

In this section, we examine the properties of bilayer fluorographene in more detail and compare them with those of monolayer fluorographene and diamond. For the study of the geometrical properties, we include also GGA calculations because they give more accurate bond lengths and angles. A summary of the geometrical and electronic properties is given in Tables II and III. The results for monolayer fluorographene compare well with previous calculations on this system. 21] As can be expected, the cell size and bond lengths and angles of bilayer fluorographene have values between those of monolayer fluorographene and diamond. Overall, the geometrical properties are close to the one of bulk diamond due to the same hybridization of the carbon atoms. 25]

The calculated $\mathrm{C}-\mathrm{C}$ bond lengths are larger than the ones in diamond. This difference can be explained from a chemical point of view as a result of the depopulation of the bonding orbitals between the carbon atoms after substantial charge transfer to the $\mathrm{F}$ atom, which is the result of the difference in electronegativity between $\mathrm{C}$ and $\mathrm{F}$ atoms.

Additionally, we have observed the shortening of the $\mathrm{C}-\mathrm{F}$ bond with increasing amount of fluorination on both sides of bilayer fluorographene. In a $3 \times 3$ supercell, the $\mathrm{C}-\mathrm{F}$ bond length varies from $1.50 \AA$ for configuration $\mathrm{B}$ to $1.43 \AA$ for configuration BBBA' A'A'. As can be seen from 
Table II] the $\mathrm{C}-\mathrm{F}$ bond length decreases even more to $1.38 \AA$ for fully fluorinated bilayer graphene. The source of this behavior can be attributed to the ionic character of the $\mathrm{C}-\mathrm{F}$ bond and a gradual transformation from $\mathrm{sp}^{2}$ to $\mathrm{sp}^{3}$ hybridization of the $\mathrm{C}$ atoms. 40]

TABLE II: Properties of single layer and bilayer fluorographene: the unit cell length $(a)$, the distances $(d)$ and angles $(\theta)$ between neighboring atoms. Distances are given in $\AA$ and angles in deg.

\begin{tabular}{ccc|cc|cc}
\hline \hline & fluorographene & \multicolumn{2}{|c|}{ bilayer } & \multicolumn{2}{c}{ diamond } \\
& & & \multicolumn{2}{c|}{ fluorographene } & \\
& LDA & GGA & LDA & GGA & LDA & GGA \\
\hline$a$ & 2.555 & 2.596 & 2.525 & 2.563 & 2.499 & 2.527 \\
$d_{\mathrm{C}-\mathrm{C}}$ & 1.553 & 1.576 & 1.541 & 1.563 & 1.531 & 1.547 \\
$d_{\mathrm{C}-\mathrm{C}^{\prime}}$ & $\mathrm{n} / \mathrm{a}$ & $\mathrm{n} / \mathrm{a}$ & 1.537 & 1.554 & 1.531 & 1.547 \\
$d_{\mathrm{C}-\mathrm{F}}$ & 1.365 & 1.382 & 1.361 & 1.377 & $\mathrm{n} / \mathrm{a}$ & $\mathrm{n} / \mathrm{a}$ \\
$\theta_{\mathrm{CCC}}$ & 110.7 & 110.9 & 110.0 & 110.2 & 109.5 & 109.5 \\
$\theta_{\mathrm{CCC}}$ & $\mathrm{n} / \mathrm{a}$ & $\mathrm{n} / \mathrm{a}$ & 108.9 & 108.7 & 109.5 & 109.5 \\
$\theta_{\mathrm{CCF}}$ & 108.2 & 108.0 & 108.9 & 108.7 & $\mathrm{n} / \mathrm{a}$ & $\mathrm{n} / \mathrm{a}$ \\
\hline \hline
\end{tabular}

The electronic properties of monolayer and bilayer fluorographene are given in TableIII we calculated the band gap of these materials together with the formation energy per atom (in contrast to previously used formation energies per fluorine atom). For comparison, the values of these quantities are also given for diamond.

The calculated band gap for monolayer fluorographene is in good agreement with previously published results when using GGA calculations. 42] Although the computed band-gap value for fluorographene is close to experimentally measured values [14, this should be seen as a coincidence. LDA and GGA calculations are known to largely underestimate the value of the band gap. The calculated band gaps are, in fact 2 times lower than more accurate results of many-electron GW calculations. 23. This apparent discrepancy is attributed to the presence of a considerable amount of defects in the experimental samples that induce midgap states (similar to defected graphane [43]).

The band gap of bilayer fluorographene is found to be larger than the one of monolayer fluorographene by approximately $1 \mathrm{eV}$. This is different from the case of hydrogenated graphene, where monolayer graphane is found to have a slightly larger band gap than bilayer graphane. 31] In fact, within LDA and GGA calculations, graphane has a larger band gap than monolayer fluorographene, 44, 45, but this observation does not apply to bilayer compounds where bilayer fluorographene surpasses bilayer graphane in the size of the energy gap. Although our calculations are not accurate enough to provide the real band gap, it is probable that this difference in the size of the band gap between monolayer and bilayer fluorographene is qualitatively correct. This follows from the fact that LDA and GGA usually produce correct trends in the variation of the band gap among similar systems. Therefore, the band gap of bilayer fluorographene has been found to be intermediate between that of fluorographene and that of bulk diamond. In this sense, diamond can be considered as the limit of multilayer fluorographene. This tendency was observed before: for theoretically proposed graphite fluoride materials involving carbon atoms with $\mathrm{sp}^{3}$ hybridization. [46]

The absolute value of the formation energy of bilayer fluorographene is smaller than the one of the monolayer, but still larger than the one of diamond. The main reason for the observed weakening of the formation energy is the drop of the ratio of the amount of fluorine atoms per carbon atom in going from monolayer to bilayer fluorographene. Overall, the qualitative image of the stability of the fluorinated materials corresponds to that of graphane and bilayer graphane in terms of the aforementioned formation energy and the presence of covalent bonds between the graphene layers, which stabilize the structure. Nevertheless, there is a large quantitative difference; the fluorinated materials are much more stable structures than the hydrogenated ones. 20.

TABLE III: Electronic band gap $E_{\text {gap }}$ and formation energy per atom $\left(E_{\mathrm{f}} /\right.$ atom $)$ of monolayer and bilayer fluorographene and diamond using LDA and GGA calculations. All the energies are given in $\mathrm{eV}$.

\begin{tabular}{lcc|cc|cc}
\hline \hline fluorographene & \multicolumn{2}{|c|}{ bilayer } & \multicolumn{2}{c}{ diamond } \\
& LDA & GGA & LDA & GGA & LDA & GGA \\
\hline$E_{\text {gap }}$ & 2.960 & 3.089 & 3.951 & 4.040 & 5.618 & 5.572 \\
$E_{\mathrm{f}} /$ atom & -1.057 & -0.944 & -0.722 & -0.593 & -0.011 & 0.132 \\
\hline \hline
\end{tabular}

The band structure of bilayer fluorographene is displayed in Fig. 4 together with a diagram of the density of states. The depicted band structure is seen to be qualitatively similar to the one of monolayer fluorographene [22] with the size of the band gap as the only obvious difference. We also calculated the effective masses of the charge carriers around the $\Gamma$-point in order to find other differences or similarities between the two materials.

The obtained effective masses of electrons, and heavy and light holes for both studied materials, can be found in Table IV It should be noted that these masses were calculated within the DFT formalism with usage of the LDA for the exchange-correlation functional, which has been shown to give reasonable results. 47]

The effective mass of charge carriers in crystalline materials usually depends on the direction in reciprocal space. Therefore, we have chosen two common directions for the hexagonal lattice at the $\Gamma$-point, namely, $\Gamma \rightarrow K$ and $\Gamma \rightarrow M$. The results for these two directions were found to be indistinguishable, and so we can conclude that the effective masses at the $\Gamma$-point are isotropic. The direction independence for the effective masses that we observe contradicts previous results in which the effective masses of monolayer fluorographene were found to be highly anisotropic. [48, Our statement about the 


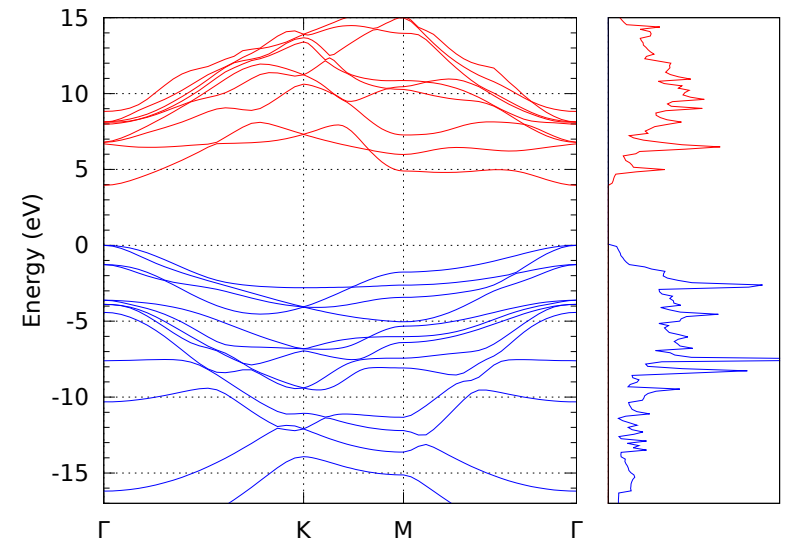

FIG. 4: Electronic band structure and density of states of bilayer fluorographene. The energies are relative to the Fermi level $\left(E_{F}=0\right)$.

isotropic character of the effective masses is supported by a direct plot of the energy value map around the $\Gamma$ point. In Fig. 5 we show a picture of the highest valence band of monolayer fluorographene. The isotropic character of this band is clearly visible close to the $\Gamma$-point. Farther away from the $\Gamma$-point an anisotropy of the surface map is visible, which can be attributed to fourth- and higher-order effects. However, these higher-order effects do not induce anisotropy in the effective mass, which is a second-order effect. Similar results are found for the light hole and the electrons.

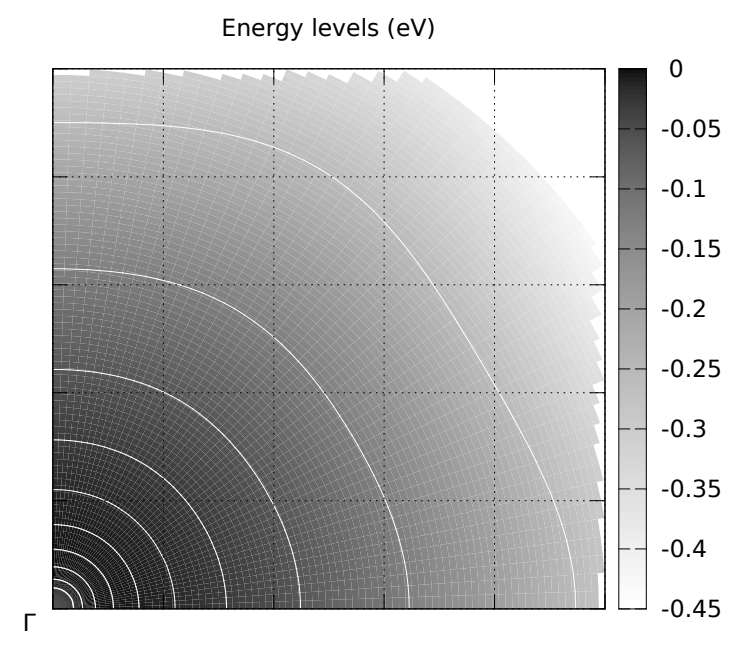

FIG. 5: Energy levels of the highest valence band of monolayer fluorographene in reciprocal space. The displayed region is a squared partition of the Brillouin zone with a side length 0.22 of the $\Gamma-M$ distance. The $\Gamma$-point is taken as the origin, and the $x$ axis directed toward the $M$-point. Contour lines are displayed for a better notion of the symmetry.

When we compare the effective masses of monolayer and bilayer fluorographene, we observe only a small dif- ference in its values. We, therefore, conclude that both materials have similar electronic properties apart from a difference in the band gap of about $1 \mathrm{eV}$.

TABLE IV: Effective masses of holes and electrons (in units of the electron mass) and the 2D Young's modulus, E, along the cartesian axes. $\mathrm{E}$ is expressed in $\mathrm{N} \mathrm{m}^{-1}$.

\begin{tabular}{lcc}
\hline \hline & fluorographene & $\begin{array}{c}\text { bilayer } \\
\text { fluorographene }\end{array}$ \\
\hline$m$ & 0.48 & 0.50 \\
$m_{h h}$ & 1.13 & 1.10 \\
$m_{l h}$ & 0.41 & 0.37 \\
$E_{x}$ & 195 & 284 \\
$E_{y}$ & 197 & 293 \\
\hline \hline
\end{tabular}

In addition to the electronic properties, we calculated the (2D) Young's modulus, $E$, for monolayer and bilayer fluorographene, which are also included in Table [IV] We followed the same approach as applied before in Ref. 22, The 2D Young's modulus of graphene is found to be $307 \mathrm{~N} \mathrm{~m}^{-1}$ shifted from the experimental value, $E_{\text {exp }}=340 \pm 50 \mathrm{~N} \mathrm{~m}^{-1}$, 49 and other theoretical values. 34] The 2D Young's modulus of fluorographene is found to be $1 / 3$ smaller in comparison to that of intrinsic graphene, whereas the obtained moduli for bilayer fluorographene reach almost the values of graphene, making these materials very strong. The values unveil the isotropic character of the Young's modulus for both compounds.

\section{CONCLUSIONS}

As an extension to previous work, 22, 31 we studied the potentially interesting case of bilayer fluorographene. We demonstrated that fluorination of bilayer graphene results in more stable structures than hydrogenation. This can be clearly observed by a comparison of the formation energies of the final structures 20 and is accentuated by the fact that the formation energy of partially fluorinated bilayer graphane is always negative, in distinct contrast to partially hydrogenated bilayer graphene. The creation of interlayer chemical bonds occurs at higher amounts of fluorination as compared with hydrogenation.

The calculated band gap of bilayer fluorographene shows a $30 \%$ increase over the one of bilayer graphane, and we also observed quantitatively significant differences between monolayer and bilayer fluorographene. From the value of the Young's modulus, we can conclude that bilayer fluorographene is substantially stronger than monolayer fluorographene and is almost as strong as graphene. 


\section{Acknowledgments}

This work is supported by the ESF-Eurocores program EuroGRAPHENE (project CONERAN) and the Flemish
Science Foundation (FWO-Vl).
[1] K. S. Novoselov, A. K. Geim, S. V. Morozov, D. Jiang, Y. Zhang, S. V. Dubonos, I. V. Grigorieva, and A. A. Firsov, Science 306, 666 (2004), http://www.sciencemag.org/content/306/5696/666.full.pdf, URL http://www.sciencemag.org/content/306/5696/ 666.abstract.

[2] K. S. Novoselov, D. Jiang, F. Schedin, T. J. Booth, V. V. Khotkevich, S. V. Morozov, and A. K. Geim, Proc. Natl. Acad. Sci. U.S.A. 102, 10451 (2005), http://www.pnas.org/content/102/30/10451.full.pdf+html, URL http://www.pnas.org/content/102/30/10451. abstract

[3] A. K. Geim and K. S. Novoselov, Nat. Mater. 6, 183 (2007), ISSN 1476-1122.

[4] N. Tombros, C. Jozsa, M. Popinciuc, H. T. Jonkman, and B. J. van Wees, Nature (London, U.K.) 448, 571 (2007), URL http://dx.doi .org/10.1038/nature06037.

[5] K. S. Novoselov, A. K. Geim, S. V. Morozov, D. Jiang, M. I. Katsnelson, I. V. Grigorieva, S. V. Dubonos, and A. A. Firsov, Nature (London, U.K.) 438, 197 (2005), ISSN 0028-0836.

[6] W. Bao, L. Jing, . J. Velasco, Jr., Y. Lee, G. Liu, D. Tran, B. Standley, M. Aykol, S. B. Cronin, D. Smirnov, et al., Nat. Phys. 7, 948 (2011), ISSN 1745-2473.

[7] C. H. Lui, Z. Li, K. F. Mak, E. Cappelluti, and T. F. Heinz, Nat. Phys. 7, 944 (2011), ISSN 1745-2473.

[8] C. L. Kane and E. J. Mele, Phys. Rev. Lett. 95, 226801 (2005), URL http://link.aps.org/doi/10. 1103/PhysRevLett.95.226801.

[9] M. Y. Han, B. Ozyilmaz, Y. Zhang, and P. Kim, Phys. Rev. Lett. 98, 206805 (2007), URL http://link.aps. org/doi/10.1103/PhysRevLett.98.206805.

[10] X. Li, X. Wang, L. Zhang, S. Lee, and H. Dai, Science 319, 1229 (2008), http://www.sciencemag.org/content/319/5867/1229.full.pc URL http://www.sciencemag.org/content/319/5867/ 1229. abstract

[11] T. Ohta, A. Bostwick, T. Seyller, K. Horn, and E. Rotenberg, Science 313, 951 (2006), http://www.sciencemag.org/content/313/5789/951.full.pdf, URL http://www.sciencemag.org/content/313/5789/ 951.abstract.

[12] E. McCann, Phys. Rev. B 74, 161403 (2006), URL http: //link.aps.org/doi/10.1103/PhysRevB.74.161403.

[13] D. K. Samarakoon and X.-Q. Wang, ACS Nano 4, $4126 \quad$ (2010), http://pubs.acs.org/doi/pdf/10.1021/nn1007868, URL http://pubs . acs.org/doi/abs/10.1021/nn1007868.

[14] R. R. Nair, W. Ren, R. Jalil, I. Riaz, V. G. Kravets, L. Britnell, P. Blake, F. Schedin, A. S. Mayorov, S. Yuan, et al., Small 6, 2877 (2010).

[15] D. C. Elias, R. R. Nair, T. M. G. Mohiuddin, S. V. Morozov, P. Blake, M. P. Halsall, A. C. Ferrari, D. W. Boukhvalov, M. I. Katsnelson, A. K. Geim, et al., Science 323, 610 (2009), http://www.sciencemag.org/content/323/5914/610.full.pdf, URL http://www.sciencemag.org/content/323/5914/ 610.abstract

[16] S. H. Cheng, K. Zou, F. Okino, H. R. Gutierrez, A. Gupta, N. Shen, P. C. Eklund, J. O. Sofo, and J. Zhu, Phys. Rev. B 81, 205435 (2010), URL http: //link.aps.org/doi/10.1103/PhysRevB.81.205435.

[17] F. Withers, M. Dubois, and A. K. Savchenko, Phys. Rev. B 82, 073403 (2010), URL http://link.aps.org/doi/ 10.1103/PhysRevB.82.073403

[18] R. Zbořil, F. Karlický, A. B. Bourlinos, T. A. Steriotis, A. K. Stubos, V. Georgakilas, K. Šafářová, D. Jančík, C. Trapalis, and M. Otyepka, Small 6, 2885 (2010).

[19] M. H. F. Sluiter and Y. Kawazoe, Phys. Rev. B 68, 085410 (2003), URL http://link .aps .org/doi/10. 1103/PhysRevB.68.085410

[20] J. O. Sofo, A. S. Chaudhari, and G. D. Barber, Phys. Rev. B 75, 153401 (2007), URL http://link.aps.org/ doi/10.1103/PhysRevB.75.153401.

[21] V. I. Artyukhov and L. A. Chernozatonskii, J. Phys. Chem. A 114, 5389 (2010), ISSN 1089-5639.

[22] O. Leenaerts, H. Peelaers, A. D. Hernández-Nieves, B. Partoens, and F. M. Peeters, Phys. Rev. B 82, 195436 (2010), URL http://link.aps.org/doi/10. 1103/PhysRevB.82.195436

[23] D. K. Samarakoon, Z. Chen, C. Nicolas, and X.-Q. Wang, Small 7, 965 (2011).

[24] H. Şahin, M. Topsakal, and S. Ciraci, Phys. Rev. B 83, 115432 (2011), URL http://link.aps.org/doi/10. 1103/PhysRevB.83.115432.

[25] J. C. Garcia, D. B. de Lima, L. V. C. Assali, and J. F. Justo, J. Phys. Chem. C 115, 13242 (2011), http://pubs.acs.org/doi/pdf/10.1021/jp203657w, URL http://pubs.acs.org/doi/abs/10.1021/jp203657w

26] A. Ueta, Y. Tanimura, and O. V. Prezhdo, J. Phys. Chem. C 116, 8343 (2012), http://pubs.acs.org/doi/pdf/10.1021/jp301856q, URL http://pubs.acs.org/doi/abs/10.1021/jp301856q

[27] K.-J. Jeon, Z. Lee, E. Pollak, L. Moreschini, A. Bostwick, C.-M. Park, R. Mendelsberg, V. Radmilovic, R. Kostecki, T. J. Richardson, et al., ACS Nano 5, 1042 (2011), http://pubs.acs.org/doi/pdf/10.1021/nn1025274, URL http://pubs.acs.org/doi/abs/10.1021/nn1025274.

[28] Z. Luo, T. Yu, K.-j. Kim, Z. Ni, Y. You, S. Lim, Z. Shen, S. Wang, and J. Lin, ACS Nano 3, 1781 (2009), http://pubs.acs.org/doi/pdf/10.1021/nn900371t, URL http://pubs.acs.org/doi/abs/10.1021/nn900371t.

[29] D. A. Dikin, S. Stankovich, E. J. Zimney, R. D. Piner, G. H. B. Dommett, G. Evmenenko, S. T. Nguyen, and R. S. Ruoff, Nature (London, U.K.) 448, 457 (2007), ISSN 0028-0836.

[30] G. Eda and M. Chhowalla, Adv. Mater. (Weinheim, Ger.) 22, 2392 (2010).

[31] O. Leenaerts, B. Partoens, and F. M. Peeters, Phys. Rev. B 80, 245422 (2009), URL http://link.aps.org/doi/ 
10.1103/PhysRevB.80.245422.

[32] T. Ohta, A. Bostwick, J. L. McChesney, T. Seyller, K. Horn, and E. Rotenberg, Phys. Rev. Lett. 98, 206802 (2007), URL http://link.aps.org/doi/10. 1103/PhysRevLett.98.206802.

[33] M. Neek-Amal and F. M. Peeters, Phys. Rev. B 83, 235437 (2011), URL http://link.aps .org/doi/10. 1103/PhysRevB.83.235437.

[34] E. Muñoz, A. K. Singh, M. A. Ribas, E. S. Penev, and B. I. Yakobson, Diamond Relat. Mater. 19, 368 (2010), ISSN 0925-9635, URL http://www.sciencedirect.com/ science/article/pii/S0925963510000166.

[35] H. Peelaers, A. D. Hernández-Nieves, O. Leenaerts, B. Partoens, and F. M. Peeters, Appl. Phys. Lett. 98, 051914 (2011), ISSN 0003-6951.

[36] J. C. Meyer, A. K. Geim, M. I. Katsnelson, K. S. Novoselov, D. Obergfell, S. Roth, C. Girit, and A. Zettl, Solid State Commun. 143, 101 (2007), ISSN 00381098, URL http://www.sciencedirect.com/science/ article/pii/S003810980700316X.

[37] J. P. Perdew, K. Burke, and M. Ernzerhof, Phys. Rev. Lett. 77, 3865 (1996), URL http://link.aps.org/doi/ 10.1103/PhysRevLett.77.3865

[38] P. E. Blöchl, Phys. Rev. B 50, 17953 (1994), URL http: //link.aps.org/doi/10.1103/PhysRevB.50.17953.

[39] H. J. Monkhorst and J. D. Pack, Phys. Rev. B 13, 5188 (1976), URL http://link.aps.org/doi/10.1103/ PhysRevB.13.5188

[40] J. O. Sofo, A. M. Suarez, G. Usaj, P. S. Cornaglia, A. D. Hernández-Nieves, and C. A. Balseiro, Phys. Rev.
B 83, 081411 (2011), URL http://link.aps.org/doi/ 10.1103/PhysRevB.83.081411

[41] M. Hasegawa and K. Nishidate, Phys. Rev. B 70, 205431 (2004), URL http://link.aps.org/doi/10. 1103/PhysRevB.70.205431.

[42] N. Lu, Z. Li, and J. Yang, J. Phys. Chem. C 113, 16741 (2009), http://pubs.acs.org/doi/pdf/10.1021/jp904208g, URL http://pubs.acs.org/doi/abs/10.1021/ jp904208g

[43] J. Berashevich and T. Chakraborty, Nanotechnology 21, 355201 (2010), URL http://stacks.iop.org/ $0957-4484 / 21 / i=35 / a=355201$.

[44] S. Tang and S. Zhang, J. Phys. Chem. C 115, 16644 (2011), http://pubs.acs.org/doi/pdf/10.1021/jp204880f, URL http://pubs.acs.org/doi/abs/10.1021/ jp204880f

[45] S. Tang and Z. Cao, Chem. Phys. Lett. 488, 67 (2010), ISSN 0009-2614, URL http://www.sciencedirect.com/ science/article/pii/S0009261410001326.

[46] Y. Takagi and K. Kusakabe, Phys. Rev. B 65, 121103 (2002), URL http://link.aps.org/doi/10. 1103/PhysRevB.65.121103

[47] M. Oshikiri, F. Aryasetiawan, Y. Imanaka, and G. Kido, Phys. Rev. B 66, 125204 (2002), URL http://link.aps . org/doi/10.1103/PhysRevB.66.125204.

[48] Y. Liang and L. Yang, MRS Online Proc. Libr. 1370, yy04 (2011), arXiv/1108.5511.

[49] C. Lee, X. Wei, J. W. Kysar, and J. Hone, Science 321, 385 (2008). 